\title{
GAUSSIAN MEASURES AND A THEOREM OF T. S. PITCHER
}

DALE E. VARBERG

1. Introduction. $B y$ a Gaussian process we shall mean a triple $\left\{X, \mathbb{B}, \lambda_{r m}\right\}$ where $X \equiv X(a, b)$ is a set of real-valued functions defined on an interval $[a, b], B$ is a Borel field of subsets of $X$ containing all sets of the form $\left\{x \in X \mid x\left(t_{0}\right)<\alpha, t_{0} \in[a, b]\right\}$ and $\lambda_{r m}$ is a Gaussian probability measure on $B$ determined by a covariance function $r$ and a mean function $m$ (how $r$ and $m$ determine such a measure is explained in [3, pp. 71-74]). The question of the equivalence or perpendicularity of two such processes (i.e., the equivalence or perpendicularity of their measures) has recently received considerable attention $[5 ; 6 ; 7 ; 9]$. It is known, for example, that a dichotomy exists. Either two such processes are equivalent or they are perpendicular (see [5]). Since a Gaussian probability measure is completely determined by a covariance and a mean function, it would seem desirable to give conditions for the equivalence (or perpendicularity) of two such measures directly in terms of the corresponding covariance and mean functions. Moreover, in the case of equivalence one would like an explicit formula for the Radon-Nikodym derivative of one measure with respect to the other. Several such results have been obtained. Of particular interest to us is a recent theorem of T. S. Pitcher (see [9, Theorem 2.3]).

THEOREM 1 (PITCher). Consider two separable Gaussian processes on $[a, b]$ with respective probability measures $\lambda_{r m}$ and $\lambda_{r 0}$ determined by the same (continuous) covariance function $r$ but with the first having mean function $m$ while the second has mean function identically zero. Suppose that $m$ is of the form $m(t)=\int_{a}^{b} r(t, s) d p(s)$ for some function $p$ of bounded variation on $[a, b]$. Then $\lambda_{r m} \sim \lambda_{r 0}$ and ${ }^{1}$

$$
\left(d \lambda_{r m} / d \lambda_{r 0}\right)(x)=\exp (1 / 2) \int_{a}^{b}[2 x(t)-m(t)] d p(t) .
$$

On the surface, this theorem appears to be of a very special nature, first of all because $m$ must be expressible in a certain way and secondly because it applies only to processes with the same covariance function. However, for a certain rather wide class of Gaussian processes we shall show that the restriction on $m$ is a very simple and natural

Received by the editors September 14, 1961.

1 We use $\sim$ between two measures to mean that the two measures are equivalent, i.e., they are mutually absolutely continuous with respect to each other. 
one. Moreover, for this class we shall remove the condition that the two processes have the same covariance function by appealing to an earlier theorem of the author [10]. This will give us the main theorem of $\$ 2$ (Theorem 4 ).

In $\$ 3$ we explore some of the consequences of Pitcher's Theorem in connection with translations of Gaussian processes, obtaining a generalization of the Cameron-Martin translation theorem for the Wiener process [2]. The relation between the author's results and some of Feldman is explored in $\$ 4$.

2. Equivalence of Gaussian probability measures. We begin with the definition of the class of probability measures with which we shall mainly be concerned.

Definition 1. We say that $\lambda_{r m} \in \mathscr{T}$ if $\lambda_{r m}$ is a Gaussian probability measure on $\{C, B\}^{2}$ determined by a covariance function $r$ and a mean function $m$ satisfying the following conditions. First, $r$ is triangular, i.e.,

where

$$
r(s, t)= \begin{cases}u(s) v(t), & s \leqq t \\ u(t) v(s), & s \geqq t,\end{cases}
$$

(A) $u(a) \geqq 0$,

(B) $v(t)>0$ on $[a, b]$,

(C) $u^{\prime \prime}$ and $v^{\prime \prime}$ exist and are continuous on $[a, b]$,

(D) $v(t) u^{\prime}(t)-u(t) v^{\prime}(t)>0$ on $[a, b]$.

Secondly, we require that $m^{\prime}$ exist and be of bounded variation on $[a, b]$.

We next state a lemma which shows that, for the triangular covariance functions described in the above definition, the apparently rather special form required for the mean function $m$ in Pitcher's Theorem becomes a simple regularity condition on $m$.

LEMMA 1. Let $r$ with factors $u$ and $v$ satisfy the conditions in Definition 1. Then the integral equation $m(t)=\int_{a}^{b} r(t, s) d p(s)$ has a solution $p$

2 Here $C=C(a, b)$ denotes the set of all continuous real-valued functions on $[a, b]$ and $B$ denotes the Borel field of subsets of $C(a, b)$ generated by sets of the form $\left\{x \in C \mid x\left(t_{0}\right)<a, t_{0} \in[a, b]\right\}$. It can be shown (see [4, pp. 401, 402]) that if $r$ and $m$ satisfy the conditions of Definition 1 we always do obtain a measure $\lambda_{r m}$ on $\{C, B\}$, i.e., the sample functions of the corresponding process may be assumed to be continuous. As examples of processes with measures belonging to $\mathfrak{T}$ we mention the Wiener (Brownian motion) process with covariance $r(s, t)=\sigma^{2} \min (s, t), \sigma^{2}>0$, and the Ornstein-Uhlenbeck process with $r(s, t)=\sigma^{2} \exp (-\beta|s-t|), \sigma^{2}>0, \beta>0$. 
of bounded variation on $[a, b]$ if and only if

(1) $m^{\prime}$ exists and is of bounded variation on $[a, b]$,

(2) $m(a)=0$ if $u(a)=0$.

Moreover, if these conditions are satisfied and if we define a function $n$ on $[a, b]$ by

$n(a)=m(a) / u(a)$ if $u(a) \neq 0$ and $n(a)=0$ if $u(a)=0$,

$n(s)=\left[v(s) m^{\prime}(s)-m(s) v^{\prime}(s)\right] /\left[v(s) u^{\prime}(s)-u(s) v^{\prime}(s)\right] \quad$ if $a<s<b$, $n(b)=0$;

then $p(s)=-\int_{a}^{s}[1 / v(t)] d n(t)$ is a solution.

Proof. Suppose that there exists a function $p$ of bounded variation on $[a, b]$ such that $m(t)=\int_{a}^{b} r(t, s) d p(s)$. Then

$$
\begin{aligned}
m(t)= & v(t) \int_{a}^{t} u(s) d p(s)+u(t) \int_{t}^{b} v(s) d p(s) \\
= & -v(t) u(a) p(a) \\
& -v(t) \int_{a}^{t} p(s) u^{\prime}(s) d s+u(t) v(b) p(b) \\
& -u(t) \int_{t}^{b} p(s) v^{\prime}(s) d s .
\end{aligned}
$$

Thus

$$
\begin{aligned}
m^{\prime}(t)= & -v^{\prime}(t) u(a) p(a)-v(t) p(t) u^{\prime}(t) \\
& -v^{\prime}(t) \int_{a}^{t} p(s) u^{\prime}(s) d s \\
& +u^{\prime}(t) v(b) p(b)-u(t) p(t) v^{\prime}(t) \\
& -u^{\prime}(t) \int_{t}^{b} p(s) v^{\prime}(s) d s .
\end{aligned}
$$

From (2.0), condition (2) of the lemma is an immediate consequence. Moreover, using (2.1) together with the fact that $u^{\prime \prime}$ and $v^{\prime \prime}$ are continuous, we can easily show that condition (1) holds.

On the other hand, suppose that conditions (1) and (2) are satisfied and let $p$ be defined as in the lemma. Then 


$$
\begin{aligned}
\int_{a}^{b} r(t, s) d p(s)= & -\int_{a}^{b}[r(t, s) / v(s)] d n(s) \\
= & -v(t) \int_{a}^{t}[u(s) / v(s)] d n(s)-u(t) \int_{t}^{b} d n(s) \\
= & -\left.v(t)[n(s) u(s) / v(s)]\right|_{a} ^{t}+v(t) \int_{a}^{t} n(s) d[u(s) / v(s)] \\
& +u(t) n(t) \\
= & v(t) n(a) u(a) / v(a) \\
& +v(t) \int_{a}^{t} \frac{v(s) m^{\prime}(s)-m(s) v^{\prime}(s)}{v(s) u^{\prime}(s)-u(s) v^{\prime}(s)} \frac{v(s) u^{\prime}(s)-u(s) v^{\prime}(s)}{v^{2}(s)} d s \\
= & v(t) n(a) u(a) / v(a)+v(t) \int_{a}^{t} d[m(s) / v(s)] \\
= & v(t) n(a) u(a) / v(a)+m(t)-v(t) m(a) / v(a) \\
= & m(t) .
\end{aligned}
$$

For later convenience, we observe that for any continuous function $y$

$$
\begin{aligned}
\int_{a}^{b} y(t) d p(t) & =-\int_{a}^{b}[y(t) / v(t)] d n(t) \\
& =\frac{n(a) y(a)}{v(a)}+\int_{a}^{b} \frac{v(s) m^{\prime}(s)-m(s) v^{\prime}(s)}{v(s) u^{\prime}(s)-u(s) v^{\prime}(s)} d\left[\frac{y(t)}{v(t)}\right] .
\end{aligned}
$$

With this lemma we may now reinterpret Pitcher's Theorem as follows.

TheOREM 2. (Same covariance, different means.) Let $\lambda_{r m}$ and $\lambda_{r 0}$ be two Gaussian probability measures on $\{C, B\}$ which belong to TT (see Definition 1) with common covariance function $r$ given by

$$
r(s, t)= \begin{cases}u(s) v(t), & s \leqq t, \\ u(t) v(s), & s \geqq t .\end{cases}
$$

Suppose further that $m(a)=0$ if $u(a)=0$. Then $\lambda_{r m} \sim \lambda_{r 0}$ and

$$
\begin{aligned}
& \quad \frac{d \lambda_{r m}}{d \lambda_{r 0}}(x) \\
& =\exp \left\{D_{1}+D_{2} x(a)+\frac{1}{2} \int_{a}^{\delta} \frac{v(t) m^{\prime}(t)-m(t) v^{\prime}(t)}{v(t) u^{\prime}(t)-u(t) v^{\prime}(t)} d\left[\frac{2 x(t)-m(t)}{v(t)}\right]\right\}
\end{aligned}
$$


where

$$
D_{1}= \begin{cases}0 & \text { if } u(a)=0 \\ -m^{2}(a) /[2 u(a) v(a)] & \text { if } u(a) \neq 0\end{cases}
$$

and

$$
D_{2}= \begin{cases}0 & \text { if } u(a)=0, \\ m(a) / v(a) & \text { if } u(a) \neq 0 .\end{cases}
$$

Proof. Combining Lemma 1 with Theorem 1, we see that $d \lambda_{r m} / d \lambda_{r 0}$ does exist and moreover, if we represent $m(t)$ as $m(t)=\int_{a}^{b} r(t, s) d p(s)$, then $\left(d \lambda_{r m} / d \lambda_{r 0}\right)(x)=\exp (1 / 2) \int_{a}^{b}[2 x(t)-m(t)] d p(t)$. But by (2.2) the latter is equal to

$$
\begin{aligned}
\exp \frac{1}{2}\left\{\frac{n(a)[2 x(a)-m(a)]}{v(a)}\right. & \\
& \left.+\int_{a}^{b} \frac{v(s) m^{\prime}(s)-m(s) v^{\prime}(s)}{v(s) u^{\prime}(s)-u(s) v^{\prime}(s)} d\left[\frac{2 x(t)-m(t)}{v(t)}\right]\right\}
\end{aligned}
$$

and this reduces to the right side of (2.3).

For convenience, we next state the earlier theorem of the author which was referred to in the introduction (see [10] for a statement and proof of this theorem in slightly different notation).

TheOREM 3. (Different covariances, same mean.) Let $\lambda_{\rho 0}$ and $\lambda_{r 0}$ be two Gaussian probability measures on $\{C, B\}$ which belong to $\mathrm{T}$ (see Definition 1) with respective covariance functions $\rho$ and $r$ given by

$$
\rho(s, t)=\left\{\begin{array}{ll}
\theta(s) \phi(t), & s \leqq t, \\
\theta(t) \phi(s), & s \geqq t,
\end{array} \quad r(s, t)= \begin{cases}u(s) v(t), & s \leqq t \\
u(t) v(s), & s \geqq t .\end{cases}\right.
$$

Then $\lambda_{\rho 0} \sim \lambda_{r 0}$ if and only if the following conditions hold:

(A) $v(t) u^{\prime}(t)-u(t) v^{\prime}(t)=\phi(t) \theta^{\prime}(t)-\theta(t) \phi^{\prime}(t)$ on $[a, b]$,

(B) $u(a)$ and $\theta(a)$ are either both zero or both nonzero. Moreover, if these conditions are satisfied

$$
\begin{aligned}
& \frac{d \lambda_{\rho 0}}{d \lambda_{r 0}}(x) \\
= & C_{1} \exp \left\{[1 / 2]\left[C_{2} x^{2}(a)+\int_{a}^{b} \frac{v(t) \phi^{\prime}(t)-\phi(t) v^{\prime}(t)}{v(t) u^{\prime}(t)-u(t) v^{\prime}(t)} d\left[\frac{x^{2}(t)}{\phi(t) v(t)}\right]\right\}\right.
\end{aligned}
$$

where 


$$
\begin{aligned}
& C_{1}= \begin{cases}\{[\phi(a) v(b)] /[\phi(b) v(a)]\}^{1 / 2} & \text { if } \theta(a)=0, \\
\{[u(a) v(b)] /[\theta(a) \phi(b)]\}^{1 / 2} & \text { if } \theta(a) \neq 0,\end{cases} \\
& C_{2}= \begin{cases}0 & \text { if } \theta(a)=0, \\
{[\phi(a) \theta(a)-u(a) v(a)] /[v(a) \phi(a) \theta(a) u(a)]} & \text { if } \theta(a) \neq 0 .\end{cases}
\end{aligned}
$$

We proceed to the main theorem of this section.

TheOREM 4. (Different covariances, different means.) Let $\lambda_{\rho \mu}$ and $\lambda_{r m}$ be two Gaussian probability measures on $\{C, B\}$ which belong to IT (see Definition 1) with respective covariance functions given by

$$
\rho(s, t)=\left\{\begin{array}{ll}
\theta(s) \phi(t), & s \leqq t, \\
\theta(t) \phi(s), & s \geqq t,
\end{array} \quad r(s, t)= \begin{cases}u(s) v(t), & s \leqq t \\
u(t) v(s), & s \geqq t .\end{cases}\right.
$$

Then $\lambda_{\rho \mu} \sim \lambda_{r m}$ if and only if the following conditions hold:

(A) $v(t) u^{\prime}(t)-u(t) v^{\prime}(t)=\phi(t) \theta^{\prime}(t)-\theta(t) \phi^{\prime}(t)$ on $[a, b]$,

(B) $u(a)$ and $\theta(a)$ are either both zero or both nonzero and if $u(a)$ $=\theta(a)=0$, then $m(a)=\mu(a)$.

Moreover, if these conditions hold

$$
\left(d \lambda_{\rho \mu} / d \lambda_{r m}\right)(x)=\left(d \lambda_{\rho 0} / d \lambda_{r 0}\right)(x-\mu) \cdot\left(d \lambda_{r, \mu-m} / d \lambda_{r 0}\right)(x-m)
$$

where the expressions on the right are given by formulas (2.4) and (2.3) respectively.

Proof. Suppose that $\lambda_{\rho \mu} \sim \lambda_{r m}$. Condition (A) follows as in the proof of Theorem $3 .^{3}$ We omit the details. To see condition (B), we note that if $\theta(a)=0$, then $1=\lambda_{\rho \mu}\{x \in C \mid x(a)=\mu(a)\}=\lambda_{r m}\{x \in C \mid x(a)=\mu(a)\}$ which implies that $\mu(a)=m(a)$ and $u(a)=0$. Similarly if $u(a)=0$, then $\mu(a)=m(a)$ and $\theta(a)=0$.

Conversely, suppose that (A) and (B) hold. We may write $\left(d \lambda_{\rho \mu} / d \lambda_{r m}\right)(x)=\left(d \lambda_{\rho \mu} / d \lambda_{r \mu}\right)(x) \cdot\left(d \lambda_{r \mu} / d \lambda_{r m}\right)(x)$ provided both derivatives on the right exist (see $[8$, p. 133]). But Pitcher has shown (see $\left[9\right.$, Lemma 1.1]) that $d \lambda_{r \mu} / d \lambda_{r m}$ exists if and only if $d \lambda_{r, \mu-m} / d \lambda_{r 0}$ exists and that

$$
\left(d \lambda_{r \mu} / d \lambda_{r m}\right)(x)=\left(d \lambda_{r, \mu-m} / d \lambda_{r 0}\right)(x-m)
$$

and the latter derivative exists by Theorem 2. Further, it is easy to show that if $d \lambda_{\rho 0} / d \lambda_{r 0}$ exists, then so does $d \lambda_{\rho \mu} / d \lambda_{r \mu}$ and that $\left(d \lambda_{\rho \mu} / d \lambda_{r \mu}\right)(x)=\left(d \lambda_{\rho 0} / d \lambda_{r 0}\right)(x-\mu)$. Now, however, Theorem 3 insures the existence of the latter derivative. This shows us that $\lambda_{\rho \mu}$ is ab-

For this proof see [10], especially \$2. The proof depends on a theorem of Baxter and, in fact, for our present theorem we need Baxter's theorem in its general form (nonzero mean) rather than a restricted version stated in [10]. However, the general form is available (see Baxter's original paper [1]). 
solutely continuous with respect to $\lambda_{r m}$ and that the Radon-Nikodym derivative has the form given in (2.5). That $\lambda_{r m}$ is absolutely continuous with respect to $\lambda_{\rho \mu}$ follows by symmetry. Thus $\lambda_{\rho \mu} \sim \lambda_{r m}$.

3. Translation theorems for Gaussian processes. Cameron, Martin (and others) have obtained an extensive transformation theory for the Wiener process. Their first result [2] was a translation theorem which we aim to generalize to a broad class of Gaussian processes. Our first theorem in this direction is as follows.

TheOREM 5.4 Let $\left\{X, B, \lambda_{r m}\right\}$ be a separable Gaussian process on $[a, b]$ with continuous covariance function $r$ and mean function $m \in L_{2}$. Let $x_{0}(t)=\int_{a}^{b} r(t, s) d q(s)$ where $q$ is of bounded variation on $[a, b]$. Then for all (B) measurable functions $F$,

$$
E^{r m}\{F(x)\}=E^{r m}\left\{F\left(x+x_{0}\right) J(x)\right\}^{s}
$$

where

$$
J(x)=\exp \left\{-(1 / 2) \int_{a}^{b}\left[2 x(t)-2 m(t)+x_{0}(t)\right] d q(t)\right\} .
$$

Proof. One may verify that for all (B) measurable functions $F$, $E^{r m}\{F(x)\}=E^{r, m-x_{0}}\left\{F\left(x+x_{0}\right)\right\}$. On the other hand, by $(2.6)$ and Theorem 1,

$$
E^{r, m-x_{0}}\left\{F\left(x+x_{0}\right)\right\}=E^{r m}\left\{F\left(x+x_{0}\right) \cdot\left(d \lambda_{r,-x_{0}} / d \lambda_{r 0}\right)(x-m)\right\} .
$$

But using (1.0), we see that $\left(d \lambda_{r,-x_{0}} / d \lambda_{r 0}\right)(x-m)=J(x)$ and this completes the proof.

Corollary 1. Let $\left\{C, B, \lambda_{r m}\right\}$ be a Gaussian process on $[a, b]$ with $\lambda_{r m} \in \mathscr{T}$ (see Definition 1), $r$ being of the form

$$
r(s, t)= \begin{cases}u(s) v(t), & s \leqq t, \\ u(t) v(s), & s \geqq t .\end{cases}
$$

Let $x_{0}$ be any function having a derivative of bounded variation on $[a, b]$ and such that $x_{0}(a)=0$ if $u(a)=0$. Then for all (B) measurable functions $F$,

$$
E^{r m}\{F(x)\}=E^{r m}\left\{F\left(x+x_{0}\right) J(x)\right\}
$$

where

4 weaker version of this result and its corollaries was obtained in the author's doctoral dissertation written at the University of Minnesota under the direction of Professor Robert H. Cameron [11].

- Erm denotes expected value on a Gaussian process with covariance function $r$ and mean function $m$. 


$$
\begin{aligned}
J(x) & =\exp \left\{D_{1}+D_{2}[x(a)-m(a)]\right. \\
& \left.-\frac{1}{2} \int_{a}^{b} \frac{v(t) x_{0}^{\prime}(t)-x_{0}(t) v^{\prime}(t)}{v(t) u^{\prime}(t)-u(t) v^{\prime}(t)} d\left[\frac{2 x(t)-2 m(t)+x_{0}(t)}{v(t)}\right]\right\}
\end{aligned}
$$

and

$$
\begin{aligned}
& D_{1}= \begin{cases}0 & \text { if } u(a)=0, \\
-x_{0}^{2}(a) /[2 u(a) v(a)] & \text { if } u(a) \neq 0,\end{cases} \\
& D_{2}= \begin{cases}0 & \text { if } u(a)=0, \\
-x_{0}(a) / v(a) & \text { if } u(a) \neq 0 .\end{cases}
\end{aligned}
$$

Proof. We apply Theorem 5 noting that $x_{0}$ is of the proper form (see Lemma 1). From the proof of Theorem 5 , we observe that $J(x)$ $=\left(d \lambda_{r,-x_{0}} / d \lambda_{r 0}\right)(x-m)$. But this derivative may be evaluated by the use of formula (2.3) which gives (2.9).

COROLlaRy 2. (Cameron-Martin translation theorem for the Wiener process. $)^{6}$ Let $\left\{C, B, \lambda_{r 0}\right\}$ be the Wiener process on $[0,1]$ with

$$
r(s, t)=\min (s, t)= \begin{cases}s, & s \leqq t \\ t, & s \geqq t .\end{cases}
$$

Let $x_{0}$ be any function having a derivative of bounded variation on $[0,1]$ and such that $x_{0}(0)=0$. Then for all (B) measurable functions $F$,

$$
E^{r 0}\{F(x)\}
$$

$$
=E^{r 0}\left\{F\left(x+x_{0}\right) \exp \left[-\int_{0}^{1} x_{0}^{\prime}(t) d x(t)-(1 / 2) \int_{0}^{1}\left[x_{0}^{\prime}(t)\right]^{2} d t\right]\right\} \text {. }
$$

Proof. We apply Corollary 1 , noting that $u(t) \equiv t, v(t) \equiv 1$ so that $D_{1}=0, D_{2}=0, v(t) u^{\prime}(t)-u(t) v^{\prime}(t) \equiv 1$, which gives the result.

4. Relation of Theorem 3 to a Theorem of Feldman. ${ }^{7}$ It is interesting to explore the connection between the results of this paper, especially Theorem 3, and some results of similar type which appear in a recent paper of Feldman [6]. Feldman considers stationary Gaus-

6 This is not quite the Cameron-Martin theorem since in that theorem the space is the set of all continuous functions vanishing at the origin rather than our $C(0,1)$. Also the Borel field $B$ has been completed with respect to the measure $\lambda_{r 0}$. Note finally that Cameron and Martin consider the process with covariance $r(s, t)=(1 / 2) \min (s, t)$ which puts an extra factor of 2 in the exponential in formula (2.10). All of these modifications could easily be made in Corollary 2 .

7 The referee suggested that this comparison be made. 
sian processes (covariance function $r(s, t)=r(s-t)$ ) with mean function identically 0 . It is well known that such covariance functions can be written as Fourier transforms, i.e., $r(\theta)=\int_{-\infty}^{\infty} \exp (-i \theta x) d F(x)$. Consider measures $\lambda_{r 0}$ and $\lambda_{\rho 0}$ determined by two such covariance functions and suppose moreover that one of them, say $r$, is the Fourier transform of $d x /\left(1+x^{2}\right)^{u}$, i.e., $r(\theta)=c \int_{-\infty}^{\infty} \exp (-i \theta x) \cdot\left(1+x^{2}\right)^{-u} d x$, where $u$ is an integer $\geqq 1$ and $c$ is a positive constant. Then Feldman gives necessary and sufficient conditions for the equivalence of $\lambda_{r 0}$ and $\lambda_{\rho 0}$ in terms of conditions on certain Fourier transforms which are related to $r$ and $\rho$ (main theorem of [6]).

The question arises as to whether Feldman's Theorem and Theorem 3 overlap. As a matter of fact, they do, but only in the case of certain Ornstein Uhlenbeck processes. It is not hard to show that the covariance functions which are both triangular (so that Theorem 3 applies) and of stationary type (so that Feldman's Theorem applies) are those which can be written in the form $r(s, t)=r(s-t)$ where $r(\theta)=\sigma^{2} \exp (-\beta|\theta|)=\left(\beta \sigma^{2} / \pi\right) \int_{-\infty}^{\infty} \exp (-i \theta x) \cdot\left(\beta^{2}+x^{2}\right)^{-1} d x, \sigma^{2}>0$, $\beta>0$. Choosing $\beta=1$ makes $r$ of the right type for the special covariance function of Feldman's Theorem. The results which are then obtained are consistent with those obtained in the author's earlier paper (see [10, Example 3]) where Ornstein Uhlenbeck processes are investigated in detail by means of what is Theorem 3 in the present paper.

\section{REFERENCES}

1. Glen Baxter, A strong limit theorem for Gaussian processes, Proc. Amer. Math. Soc. 7 (1956), 522-527.

2. R. H. Cameron and W. T. Martin, Transformations of Wiener integrals under translations, Ann. of Math. 45 (1944), 386-396.

3. J. L. Doob, Stochastic processes, Wiley, New York, 1953.

4. - Heuristic approach to the Kolmogorov-Smirnov theorems, Ann. Math. Statist. 20 (1949), 393-403.

5. Jacob Feldman, Equivalence and perpendicularity of Gaussian processes, Pacific J. Math. 8 (1958), 699-708.

6. - Some classes of equivalent Gaussian processes on an interval, Pacific J. Math. 10 (1960), 1211-1220.

7. Jeroslav Hajek, On a property of normal distributions of any stochastic process, Selected Translations in Mathematical Statistics and Probability 1 (1961), 245-252.

8. Paul R. Halmos, Measure theory, Van Nostrand, New York, 1950.

9. T. S. Pitcher, Likelihood ratios of Gaussian processes, Ark. Mat. Band 4, Häfte 1 (1960), 35-44.

10. Dale E. Varberg, On equivalence of Gaussian measures, Pacific J. Math. 11, No. 2 (1961), 751-762.

11. - Some Radon-Nikodym derivatives associated with stochastic processes, Thesis, University of Minnesota, 1959.

HAMLINE UNIVERSITY 